\title{
Der Urogenitalkrebs in seiner Bedeutung für das Krebsproblem.
}

Vortrag, gehalten im Komitee für Krebsforschung zu Berlin am 6. Dezember 1902.

Von

C. Posner.

M. H.! Wer es unternimmt, in diesem Kreise, der sich die Erforschung und Bekämpfung der Krebskrankheit im weitesten Umfange zur Aufgabe gestellt hat, vom Krebs eines Organes oder Organsystems zu sprechen, muss nachweisen, dass gerade an dem gewählten Beispiel eine grosse Zahl der schwebenden Fragen Klärung oder doch wenigstens Beleuchtung finden kann, dass durch die Einzelbetrachtung das Allgemeinverständnis eine Förderung erfährt. Auf zwei Wegen, wenn sie auch oft noch so sehr voneinander abzuweichen scheinen, suchen wir das gemeinsame Ziel: wir gehen ins Breite, indem wir uns bemühen, durch Sammlung möglichst reichhaltigen Materials generelle Folgerungen zu ziehen - wir dürfen aber darüber nicht vergessen, in die Tiefe zu dringen, die uns durch detaillierte Prüfung, durch Erforschung und Feststellung auch des scheinbar Geringsten doch erst die zuverlässigen Grundlagen unserer Erkenntnis zu erschliessen verspricht.

Die Erkrankungen des Urogenitalapparates haben sich bereits für manche anderen Gebiete der Pathologie besonders lehrreich erwiesen. Ich erinnere daran, dass z. B. für die Tuberkulose hier mit seltener Klarheit die Infektionswege zu tage traten, seitdem Cohnheim zuerst den Begriff der "Ausscheidungstuberkulose" fixiert hat. Ich lenke Thre Aufmerksamkeit auf die gerade hier erfolgreich studierten Fragen der Selbstinfektion; auf die Vorgänge bei Bildung der Konkremente; endlich auf die so leicht und sicher zu verfolgenden Wirkungen der Ansteckung durch die verschiedenen Formen der venerischen Erkrankungen. Und für die Probleme der Krebskrankheit treffen hier ohne Zweifel mancherlei Bedingungen zusammen, die leichter wohl als an manchen anderen Organen in Bezug auf ihre Wirksamkeit geprüft werden können: die häufige Gelegenheit zur An- 
steckung, die am Geschlechtsapparat besonders nahe liegende Möglichkeit einer Verirrung von Keimanlagen, die vielen traumatischen und entzündlichen Reize, die Veränderungen schliesslich, welche in dem wesentlichsten Ausscheidungsprodukte des Körpers, dem Harn, vor sich gehen.

Frühe schon hat sich namentlich die Aufmerksamkeit der Chirurgen der Frage zugewandt, ob etwa durch den Geschlechtsverkehr selbst eine U ebertragung des Krebses stattfinden könne. Durchmustert man die vorliegende Literatur, wie sie unser Mitglied, Herr $\mathrm{Behla}$, in verdienstlicher Weise zusammengestellt hat ${ }^{1}$ ), prüft man weiter die Sammelforschung unseres Komitees ${ }^{2}$ ), so wird man, wie mich bedünkt, bekennen müssen, dass die Ausbeute in dieser Hinsicht nicht gerade als erheblich bezeichnet werden kann - namentlich wenn man die ungeheuere Verbreitung des Carcinoms des Uterus bezw. der Portio vaginalis bedenkt. Unsere Sammelforschung berichtet über 2330 Fälle von Krebs der weiblichen, über 107 Fälle von Krebs der männlichen Genitalien, und es wird freilich dabei 38 mal bei Weibern, 4mal bei Männern (3 Peniskrebse, 1 Hodenkrebs) die Vermutung einer Ansteckung durch den Ehegatten ausgesprochen. Indes ist dabei nicht ausgedrückt, ob in diesen scheinbaren Ansteckungsfällen es sich wirklich beiderseits um Sitz des Carcinoms an den Geschlechtsorganen gehandelt habe; meist scheint vielmehr ein Cancer à deux anderer Art vorzuliegen. So verhält es sich z. B. in einem von Herrn $\mathrm{B}$ ehla mitgeteilten Falle ${ }^{3}$ ), in dem die Frau an Gebärmutter-, der Mann an Nierenkrebs litt, so auch in dem von ihm zitierten Falle von Berger (Mann Peniscarcinom, Frau Krebs der rechten Ohrmuschel). Und dringt man noch etwas tiefer in die Statistik ein, so sieht man z. B. unter 773 Fällen von Gebärmutterhalskrebs 11 mal eine Ansteckung durch den Gatten vermutet - aber diese Zahl 11 verliert meines Erachtens erheblich an Bedeutung, wenn man erwägt, dass in 13 Fällen Syphilis, in nicht weniger als 24 Abort als Ursache angeschuldigt wird. Ich glaube nicht, dass jemand in letzterem Verhältnis mehr als ein zufälliges, höchstens ein prädisponierendes Moment erblicken wird; und so meine ich auch, wird sich mancher Fall scheinbarer Ansteckung ungezwungen als ein angesichts der ungeheueren Verbreitung des Krebses wohl begreifliches Zusammentreffen deuten lassen. Herr Behla exemplifiziert auf den Diabetes der Ehegatten - mir scheint dieses Beispiel, nach allem, was hierüber bekannt ist, eher ge gen als für die Annahme einer Infektion ver-

1) Die Carcinomliteratur, von Robert Behla. Berlin 1901, Verlag von Richard Schoetz.

2) Bericht über die vom Komitee für Krebsforschung am 15. Oktober 1900 erhobene Sammelforschung. Jena, Gustav Fischer, 1902 (Klin. Jahrbuch).

3) Zeitschr. f. Medizinalbeamte, 1900 , S. 164. 
wertbar zu sein. Jedenfalls wird man für derartige Beobachtungen vor allen Dingen Yorkommen an don boidorsoitigen Gropitalorganen und genaue Angaben über Entstehung und Verlauf fordern müssen. Immer wieder wird man ganz speziell auf den bekannten Fall von Tross ${ }^{1}$ ) verwiesen, in welchem in der Tat Penis- und Portiocarcinom aufeinander folgten; ganz so zwingend, wie Behla meint, scheint mir auch dieser Fall nicht - denn die histologische Uebereinstimmung dieser Krebse ist wohl verständlich, auch ohne dass man den einen für einen Abkömmling des anderen zu halten braucht. Es handelt sich in der vorliegenden Literatur im ganzen um etwa 30 Fälle (nach Behla); wie viele von ihnen der notwendigen, strengen Kritik Stich halten, möchte ich dahingestellt sein lassen.

Im übrigen erklärt $\mathrm{Cz}_{\mathrm{z}} \mathrm{rny} \mathrm{y}^{2}$, gewiss mit Recht, dass die Eventualität einer Ansteckung ja dadurch verringert werde, dass ein Coitus mit gebärmutterkrebskranken Frauen immerhin wohl nicht allzu häufig sei. Man kann als weiteren Grund für die Seltenheit der Uebertragung auch anführen, dass, wenn überhaupt die hier vorausgesetzte Ueberimpfung von Krebs angenommen werden darf, aller Wahrscheinlichkeit nach hierzu eine, wenn auch noch so unbedeutende Verletzung, Exkoriation o. dgl. die Vorbedingung bilden muss.

Letztere Bedingung wird jedenfalls erfüllt bei den hier sich anschliessenden Fällen von Kontaktinfektion und Implantation. Versteht man unter Kontaktinfektion zunächst das unmittelbare Uebergreifen eines Krebsherdes auf eine gegenüberliegende Haut- oder Schleimhautstelle - also ohne Vermittelung der Blut- oder Lymphbahnen, welche die eigentliche Metastase übertragen - so ist namentlich die gynäkologische Literatur reich an wohlbeglaubigten Beispielen Es kommt bei Krebs der Portio vor, dass anliegende Stellen der Vagina krebsig erkranken, es pflanzt sich das Carcinom eines Labiums auf das andere fort ${ }^{3}$ ). Für die Harnblase hat namentlich Albarran ${ }^{4}$ ) wiederholt beschrieben, wie ein breiter, pilzförmig sich lagernder Tumor auf den mit ihm in Berührung kommenden Teilen der Blasenwand Neubildungen ganz gleichen Charakters erzeugt, welche von dem ursprünglichen Herd durch weite Strecken in jeder Hinsicht gesunden Gewebes getrennt sind. Die Fälle der Art, für welche wir zunächst immer einen entzündlichen Reiz, eine oberflächliche Wunde der Schleimhaut annehmen, in denen das Krebsgewebe zur Keimung gelangt, sind uns verständlich, wenn wir nur

1) Beiträge zur Frage der Uebertragbarkeit des Carcinoms. I.-D. Heidelberg 1887. Vgl. hierzu C z erny, Warum dürfen wir die parasitäre Theorie der bösartigen Geschwülste nicht aufgeben? Beitr. z. klin. Chir., Bd. 25.
2) I. C. B. Fälle von Thorn, Centralbl. f. Gynäkol., 1894. Weitere Literatur bei Behla, l. c.
4) Les tumeurs de la vessie. Paris 1893. 
iberhaupt die durch vielerlei Experimente und Erfahrungen bewiesene Lebensfähigkeit der in andere Teile des gleichen Organismus gelangenden Krebszellen anerkennen.

Wesentlich schwieriger für unsere Vorstellung liegt die Implantation in entferntere Organe. James Israel erwähnt in seiner trefflichen Monographie über die chirurgischen Krankheiten der Niere 3 Fälle von "Zottenkrebs", die ihren primären Sitz im Nierenbecken hatten, aber auf dem Harnwege sekundäre Wucherungen in der Blase hervorriefen ${ }^{1}$ ). Es ist natürlich, wie Israel selber hervorhebt, schwierig, in solchen Fällen die Metastase auf dem Blut- oder Lymphwege ganz auszuschliessen, indes spricht der Verlauf, namentlich das Auftauchen der Tumoren gerade an der Mündungsstelle des Harnleiters doch sehr zu Gunsten der obigen Annahme. Sie wird weiter erhärtet durch einige andere Beobachtungen von $B u s e^{2}$ ) und $R$ eynès ${ }^{3}$, die das Gleiche von anscheinend gutartigen, später teilweise krebsig gewordenen Papillomen des Nierenbeckens gesehen haben.

Auch für den weiblichen Geschlechtsapparat ist Aehnliches wiederholt behauptet worden: es soll von der Portio aus eine Dissemination von Krebskeimen auf das Innere des Uteruscavums stattfinden, welche sich dadurch dokumentiert, dass dort ganz vereinzelte Inseln von Carcinom auftreten, die genau in ihrem histologischen Bau dem primären Tumor entsprechen. Winter hat gezeigt, dass die Fälle der Art auch anderer Deutung fähig und nicht streng beweisend sind ${ }^{4}$ ). Eine ganz nenerlich ron $\mathrm{K}$ unze ${ }^{5}$ ) mitgeteilte gleichartige Beobachtung scheint allerdings, soweit ich mir ein Lrteil gestatten darf, ziemlich einwandfrei.

Es muss für alle zuletzt erwähnten Fälle nochmals das ganz besondere allgemein-pathologische Interesse betont werden; sie stehen in Analogie zu den wiederholt beschriebenen "Schluckcarcinomen", d. h. zur Implantation von Krebspartikeln aus der Speiseröhre auf die Magenschleimhaut, wie solche noch neuerdings von Fütterer ${ }^{6}$ ) unter Hinweis auf frühere ähnliche Beobachtungen anderer Autoren behauptet worden ist.

Unzweifelhaft feststehend ist im Gegensatz hierzu die Tatsache, dass gerade beim Genitalkrebs des Weibes das "Impfcarcino m" im Anschluss an Operationen nicht allzu selten erscheint. Ich brauche

1) J. Is ra e l, Chirurg. Klinik der Nierenkrankheiten. Berlin, A. Hirschwald, 1902 , S. 451,532 ff.

2) Virchows Arch., Bd. 164.

3) Ann. des Mal. des Org. génito-urin., 1902, Décbr. (Ber. über d. Jahresvers. der Ass. urol. franç.).

4) Veits Handbuch d. Gynäkol., Bd. 3, H. 2.

5) Münchener med. Wochenschr., 1901 (Jen. med.-natw. Gesellsch.).

6) Die Aetiologie des Carcinoms. Wiesbaden, J. F. Bergmann, 1901. 
an dieses Ereignis hier nur zu erinnern, nachdem erst ganz vor kurzem von autoritativster Seite $\left[0 \mathrm{lshausen}^{1}\right.$ ) über einen solchen Fall berichtet worden ist.

Handelte es sich in den bisher erwähnten Fällen wesentlich um Verschleppung lebenden, ausgebildeten Krebsgewebes im Organismus und dessen Wucherung an anderen Stellen, so ist man gerade beim Urogenitalsystem schon aus entwickelungsgeschichtlichen Gründen gern geneigt, für die Entstehung neuer Tumoren auch an verirrte oder versprengte Keimgewebe im Sinne der Cohnheimschen Theorie zu denken. Namentlich wird man ja auf diese förmlich hingewiesen, wo es sich um Neubildungen handelt, die schon im kindlichen Alter, ja sogar, wie in C. Weigerts bekanntem Falle, intrauterin erscheinen können. Durchmustert man ältere Statistiken von Nierenkrebs, so ist ja die Beteiligung der ersten Lebensjahre ganz erstaunlich; so wollte z. B. Rohrer unter 96 Fällen 37mal, also in fast 40 Proz., Nierenkrebs bei Kindern unter 10 Jahren gesehen haben; und ähnlich lauten die Zahlen von Ebstein und Lach$\operatorname{man} n^{2}$ ).

Hier hat sich nun unser Standpunkt in den letzten Jahren insofern wesentlich verrückt, als wir heute zwar den angeborenen, auch den malignen Charakter vieler der in Rede stehenden Neubildungen anerkennen - nicht aber den carcinomatösen. Seitdem Grawitz zuerst auf das Vorkommen von Nebennierengewebe in den Nierentumoren hingewiesen hat, sind durch fortlaufende Untersuchungen - ich erwähne nur die Arbeiten von Birch-Hirschfeld, v. Han semann, Lubarsch - eine grosse Zahl von Typen erkannt worden, die wohlcharakterisirt sind und mit dem eigentlichen Carcinom nichts. gemein haben: die Hypernephrome, die malignen Endotheliome, maligne Adenome, Teratome u. a. m. Schliesst man alle diese Formen, die hier nicht zur Diskussion stehen, aus, so bleibt für das Kindesalter nur ein sehr geringer Prozentsatz ron echten Carcinomen zurück - nicht grösser als in anderen Organen: und deren Ableitung im Cohnheimschen Sinne schwebt tatsächlich in der Luft.

Für die Geschlechtsorgane, beim Manne wie beim Weibe, liegen die Verbältnisse wohl ziemlich ähnlich; auch hier kommen gelegentlich in früher Kindheit sich entwickelnde Geschwïlste zur Beobachtung, auf die embryonale Genese der Carcinome aber fällt kein neues Licht.

Erwähnen aber möchte ich immerhin hier eine Beobachtung von Ehrlich ${ }^{3}$ ), die, wenn auch unter sehr komplizierten und abnormen

1) Deutsche med. Wochenschr., 1902, Nr. 42.

2) Vgl. Senator, Die Krankheiten der Niere in Nothnagels Sammelwerk, 1902 .

3) Gallertkrebs der ekstrophierten Blase. Bruns Beitr., Bd. 30. 
Posner, Urogenitalkrebs in seiner Bedeutung für das Krebsproblem.

Verhältnissen, doch die Möglichkeit der Bildung von Carcinom aus verlagertem Epithel illustriert: er sah auf einer ekstrophierten Blase einen Kolloidkrebs sich entwickeln, der alle Charaktere des Dickdarmkrebses trug und sicherlich nur durch Anwesenheit echten Darmepithels in der Schleimhaut erklärt werden kann.

Im ganzen etwas mehr vermögen wir auszusagen über die $\mathrm{Be}$ deutung der chronischen Reize“, entzündlicher sowohl als tranmatischer und chemischer.

Von der chronischen Entzündung der Urogenitalschleimhaut wissen wir schon seit einer Reihe von Jahren, dass sie hier nahezu konstant zu einer epidermalen Metaplasie (Pachydermie, Verhornung) führt ${ }^{1}$ ). Insbesondere gilt dies für Urethra und Vagina; doch auch von der Blase ${ }^{2}$, dem Nierenbecken, auch dem Uterus ${ }^{3}$ ) sind uns solche „Leukoplasien" wohlbekannt. Die Akten über das Verhältnis von Carcinom und Leukoplasie sind ja nun freilich keineswegs geschlossen; an anderen Organen, namentlich am Kehlkopf, wird bekanntlich von vielen Autoren eine nahe Verwandtschaft angenommen ${ }^{4}$ ). Fine gewisse histologische Aehnlichkeit ist nicht zu leugnen; tiefgreifende Epithelzapfen, Hornperlen u. s. w. finden wir bei der einfachsten Entzïndung, wie sie z. B. das Bestehen eines Scheidenvorfalles begleitet; auch dem geübtesten Untersucher fällt es keineswegs immer leicht, aus dem mikroskopischen Bilde allein zu erkennen, ob er es mit einer einfachen Epidermisierung oder mit einem beginnenden Cancroid zu tun hat.

Die klinische Erfahrung spricht nun zum mindesten nicht gegen eine gewisse Beziehung der Metaplasie zum Carcinom. Hallé z. B. fand unter 20 Fällen von Harnröhrencarcinom 12mal das Vorangehen

1) Vgl. z. B. Posner, Unters. über Schleimhautverhornung. Virch. Arch., Bd. 118. Daselbst auch ältere Literatur.

2) Z. B. W end le r, Mitt. a. d. Grenzgebieten, Bd. 6, 1900. - Pollack, Arb. aus dem pathol. Inst. zu Posen, hrsg. v. Lu barsch, Wiesbaden, Bergmann, 1902. - Ich benutze die Gelegenheit, eine irrtümliche Auffassung über meine Stellung zur Metaplasiefrage zu berichtigen, die u. a. in Wendler s zitierter Arbeit sich findet. Als ich die Ergebnisse meiner Untersuchungen zuerst auf der NaturforscherVersammlung in Köln vortrug, warnte Herr $\mathrm{M}$ a $\mathrm{r} \mathrm{ch}$ and vor Verallgemeinerungen, indem er auf einen Fall von Verhornung des Blasenepithels hinwies, die seiner Meinung nach durch Hineinwuchern von Epidermis durch eine Blasenfistel, nicht aber durch Metaplasie entstanden sei. Ich acceptierte selbstrerständlich diese Deutung des trefflichen Beobachters und erwähnte den Fall in meiner Pachydermie-Arbeit in seinem Sinne. Später hat Herr M a rchand selber seine erste Auffassung geändert und auch für diesen Fall die Metaplasie angenommen; danach habe ich natürlich auch keine Veranlassung mehr, an seiner ersten Deutung festzuhalten, stelle also hier fest, dass ich nicht, wie Wendler tut, als ein Verteidiger der Ueberwucherungstheorie bezeichnet werden möchte, um so weniger, als ja gerade mein e Arbeiten zuerst den umfassenden Nachweis erbracht haben, dass Schleimhäute der verschiedensten entwickelungsgeschichtlichen Herkunft der epidermidalen Metaplasie fähig sind.

3) v. Rosthorn u. a.

4) Siehe hierzu: B. Fraenkel, Metaplasie und Krebs des Kehlkopfes. Arch. f. Laryngol., 1902. 
einer gonorrhoischen Striktur; auch in der neueren Kasuistik wird immer wieder auf diesen Zusammenhang hingewiesen. Marchands oben (s. d. Anm.) erwähnter Fall lehrt ebenfalls, dass sich an Leukoplasie der Blase Hornkrebs anschliessen kann; Albarrand) spricht sich auf Grund von 4 Beobachtungen im gleichen Sinne aus, ebenso neuerdings $W$ endler ${ }^{2}$ ) und $\operatorname{Lubarsch}{ }^{3}$ ).

Besonders deutlich tritt ein Zusammenhang von Krebs und Har $\mathrm{n}$ röhrenfistel hervor. Wie auch in anderen Fistelgängen - z. B. in solchen, die zu Knochenladen führen - sich Krebs mit Vorliebe etabliert, so ist auch beim Penis- bezw. Harnröhrenkrebs das Gleiche verhältnismässig häufig notiert, und öfter dabei mit Sicherheit der Gang der Ereignisse zu verfolgen: chronische Gonorrhöe oder Trauma, dann Striktur, periurethrale Infiltration, Fistelbildung, Fistelkrebs. Solche Beobachtung hat kürzlich noch $\mathrm{Koenig}^{4}$ ) mitgeteilt; und ein Fall meiner eigenen Praxis, von meinem früheren Assistenten, Herm Dr. Lipman-Wulf, ausführlich beschrieben ${ }^{5}$ ), lässt das Gleiche mit aller Bestimmtheit behaupten.

Schwieriger liegt die Frage, ob auch der durch die Anwesenheit von K on krementen gesetzte Reiz als ein disponierendes Moment aufgefasst werden darf. An sich wäre die Wahrscheinlichkeit eines Zusammenhanges nicht gerade gering; für die Gallenblase wird er von vielen Autoren mit Bestimmtheit angenommen $\left.{ }^{6}\right)$. Für Blase und $\mathrm{Niere}$ fehlt es an stringentem Beweismaterial - in J. Israels sorgsam geführten Krankengeschichten finde ich z. B. unter 8 Carcinomen des Nierenbeckens keinen Stein, unter 74 Steinen keinen Tumor: auch persönlich hat Herr I s r a e l mir wiederholt, dass er einen etwaigen Zusammenhang jedenfalls als sehr locker ansehen müsse. Die meisten Autoren erwähnen freilich Steinbildung als zu Krebs disponierend bestimmt verwertbare Tatsachen aber kann ich nicht entdecken und auch nicht aus der eigenen Erfahrung angeben.

Besonders gut beglaubigt - und in dieser Hinsicht nahezu ohne Beispiel in der gesamten Pathologie - ist das Auftreten des Carcinoms, freilich nicht der Schleimhaut, sondern der äusseren Bedeckung der Geschlechtsteile, nach einer ganz bestimmten Reizung che mis cher Art: des Teer-, Paraffin- und Schornsteinfegerkrebses. An der spezifischen Bedeutung dieser zumeist am Scrotum lokalisierten Krebse kann wohl kein Zweifel bestehen. Der Schornsteinfegerkrebs speziell ist in seiner ătiologischen Bedeutung, d. h. in seiner Beziehung. zu den Verbrennungsprodukten der Steinkohle, völlig sicher gestellt

1) 1. c.

2) l. c.

3) Arb. aus dem pathol. Inst. in Posen. Wiesbaden, Bergmann, 1902.

4) Monatsh. f. Urologie, 1901.

5) Verh. d. Berliner med. Ges., Dezbr. 1902.

6) Vgl. Fütterer, l. c. 
seitdem Ba um ${ }^{1}$ ), der die Krankheit zuerst in Deutschland sah, nachgewiesen hat, dass die Affektion in Hannover erst auftrat, als man von dem bis dahin geübten Heizen mit Holz zur Verwendung der Kohlen überging. In diesen Fällen kommt es zuerst zu Verhärtungen und Warzen der Haut, an die sich dann erst die Krebsbildung anschliesst. Sehr ähnlich sind die Verhältnisse bei dem namentlich ron Tolkmann ${ }^{2}$ ) und Tilmanns ${ }^{3}$ ) studierten Paraffinkrebs; hier geht allerdings keine warzenartige Verdickung, sondern eine eigentiimliche Starrheit der Haut mit schwärzlicher Färbung vorauf.

Von einer Beteiligung der B $\backslash$ a s e wird in derartigen Fällen nichts gemeldet. Ich verdanke aber persönlicher Mitteilung des Herrn Kollegen H. Goldschmidt-Berlin die Kenntnis eines einschlägigen, sehr bemerkenswerten Falles, in welchem bei einem Teerarbeiter sich der typische $\mathrm{Skrotalkrebs}$ entwickelt hatte (2mal, von Herrn v. Bergmann und von Herrn Sonnenburg operiert), ausserdem aber Strangurie und Hämaturie eintraten, als deren Ursache sich bei der kystoskopischen Untersuchung ein $\mathbf{B l}$ as entumor ergab, welcher, gerade wie die Hautkrebse, schwärzlich gefärbt erschien und seinem ganzen Verhalten nach unzweifelhaft als Carcinom angesprochen werden musste.

Es führt uns dieser Fall direkt zu den das grösste Aufsehen verursachenden Beobachtungen über das Vorkommen von Blasentumoren bei Anilinarbeitern, welche Rehn ${ }^{4}$ ) zuerst auf dem Chirurgenkongress 1897 bekannt gab. Anfänglich war man wohl geneigt, den ursächlichen Zusammenhang zu bezweifeln, vielmehr hierin nur ein Spiel des Zufalls zu erblicken; indes haben sich doch durch die Mitteilungen von Leichtenstern ${ }^{5}$ ) und Wendler ${ }^{6}$ ) die Fälle soweit gemehrt, dass man nun, namentlich wenn man sich des ziemlich häufigen Vorkommens von Blutharnen bei den Anilinarbeitern erinnert, den Kausalnexus wohl nicht mehr bestreiten kann. Der letztgenannte Autor konnte 1900 immerhin 6 solcher Fälle zusammenstellén - davon 4 Carcinome, 2 Sarkome; es scheint mir dabei besonders beachtenswert, dass, bei gleicher Schädlichkeit, bald diese, bald jene Form der malignen Neubildungen erzeugt wird.

Persönlich habe ich einen solchen Fall nicht gesehen, wohl aber, was hier immerhin anzureihen ist, einen Blasenkrebs bei einem $\mathrm{N}$ a phtholarbeiter, bemerkenswerth noch dadurch, dass der Bruder des Patienten, der in der gleichen Fabrik arbeitete, an Blasenstein litt. Eine Erklärung des Zusammenhanges zu geben, sind wir freilich ausser stande; eine grosse Anzahl von Tierversuchen, die ich gemeinsam mit Herrn R. Huldschiner im Laboratorium des Herm Geh.

1) Vgl. Fütterer, l. c.

2) Ueber Teer- und Russkrebs. Verh. d. D. Ges. f. Chirurgie, 1874.

3) D. Zeitschr. f. Chirurgie, 1880.

4) Blasengeschwülste bei Anilinarbeitern, Arch. f. klin. Chir., Bd. 50.

5) Deutsche med. Wochenschr., 1900.

6) l. c. 
Rat Liebreich anstellte ${ }^{1}$ ) und die darauf abzielten, etwa eintretende Veränderungen des Harns oder der Blase zu studieren, sind eigentlich ganz ohne greifbares Resultat geblieben - die einzige gelegentlich beobachtete Veränderung des Harnapparats bestand im Vorkommen von Oxalurie und vereinzelten Nierenblutungen. Mehrmals fanden wir Ausscheidung von Anilin in Substanz durch den Harn. Es war im Uebrigen auffallend, dass selbst bei sehr lange fortgesetzter Dauer oder bei immer neu wiederholter Inhalation von Anilin, Toluidin und Nitrobenzol keinerlei spektroskopisch nachweisbare Blutzersetzung eintrat. Die Tiere gingen vielmehr unter den Erscheinungen von Anämie, allgemeiner Prostration, Coma mit Konvulsionen zu Grunde.

Von anderen Reizen, die die Blase treffen und hier carcinomatöse Neubildungen hervorrufen sollen, muss ich noch zweier Par a s it en gedenken, die in diesem Sinne beschuldigt sind: der Bilharzia und der Coccidien. Erstere führt bekanntlich oft zu Blasenblutungen und Wucherungen - inwieweit diese den Namen echter Krebse verdienen, muss ich dahingestellt sein lassen. In einem Falle Al ba r ran s ${ }^{2}$ ? fand sich jedenfalls Koinzidenz - im Gewebe eines Blasenkrebses liessen sich Bilharzia-Eier nachweisen. Derselbe Autor gibt an, auch wiederholt in Blasencarcinomen, wie auch im Krebs anderer Organe Coccidien oder Psorospermien angetroffen zu haben ${ }^{3}$ ); es ist wohl nicht ganz ausgeschlossen, dass es sich dabei, mitunter wenigstens, um Erfüllung von Zellen mit R ussellschen oder Plimmerschen Körperchen gehandelt haben kann. Eines Urteils ïher das Vorkommen der letztgenannten Gebilde, ihr Wesen und ihre Bedeutung möchte ich mich angesichts des geringen bisher hier vorliegenden Materials rorläufig enthalten. Ich möchte nur betonen, dass, wenn einmal deren pathogenetis che Bedeutung sicher gestellt werden sollte, die bisher mitgeteilten Tatsachen natürlich nichts von ihrer Bedeutung verlieren würden - sie würden dann immer noch als unentbehrliche ätiologische Momente anzusprechen sein.

Das direkte Trauma - Schlag, Stoss, Kontusion - spielt, soweit ich die Literatur übersehe, höchstens für das Peniscarcinom eine Rolle; hier stellte namentlich Englisch4) eine ganze Anzahl von Fällen zusammen, in denen die Entstehung der Krankheit auf eine solche Veranlassung zurückgeführt wurde. Sonst ist in dieser Richtung nur zu erwähnen, dass manche Autoren geneigt sind, Geburtsverletzungen als eine Ursache des Uteruskrebses anzusprechen ${ }^{\%}$.

Allgemein anerkannt ist aber, dass gewisse Reize, gerade in Blase und Harnoöhre, papilläre Exkreszenzen unzweifelhaft gutartiger

1) Vgl. Huldschiner, Münchener med. Wochenschr., 1900.

2) Albarran et Bernard, Arch. de Méd. expér., T. 9.

3) Les tumeurs de la vessie. Paris 1893.

4) Englisch, Wiener med. Wochenschr., 1902.

5) Cullen, Cancer of the uterus. Baltimore 1900 . 
Natur erzeugen können; ich erinnere an kleinste, zottige Wucherungen in der Umgebung des Blasenhalses, an die Karunkeln der weiblichen Urethra, die jedem, der viel kystoskopiert oder endoskopiert hat, wohlbekannt sind. Die Frage ist nun: können solche ursprünglich gutartige Tumoren auch, wie man sagt, krebsig entarten? Ich muss für die Harnblase voraufschicken, dass es keineswegs leicht ist, von einem Tumor mit Bestimmtheit auszusagen, ob er gutartig oder bösartig ist. Nach dem kystoskopischen Bilde, selbst nach der operativen Autopsie ist diese Diagnose durchaus nicht stets mit völliger Sicherheit zu stellen; insbesondere beweist bekanntlich die Anwesenheit noch so schön ausgebildeter Papillen nichts, da eben alle Wucherungen solche zu tragen pflegen. Ich erinnere mich - leider - so manchen Falles, in dem man noch bei der Operation glauben durfte, es mit einem ganz beschränkten, benignen Tumor zu tun zu haben, während dann doch die mikroskopische Untersuchung die carcinomatöse bezw. sarkomatöse Natur feststellte. Es hat sich allmählich unsere früher wohl etwas zu optimistische Auffassung der Blasengeschwülste gewandelt, und es ist bezeichnend, dass z. B. Albarran unter 88 selbst untersuchten Fällen durch minutiöse Zerlegung in Serienschnitte 68mal die Diagnose: Krebs stellen musste. Man kann danach - wie dies z. B. auch L u barsch tut - in allen Fällen, in denen ursprünglich anscheinend gutartige Geschwiilste in krebsige sich zu verwandeln scheinen, mit ziemlich gutem Grunde behaupten, dass dann die Geschwulst von allem Anfang an bösartig war, und man es eben nur mit einer bei Blaśengeschwülsten gar nicht seltenen, sehr langsamen Entwickelung zu tun gehabt hat. So dürften sich z. B. Kümmells Beobachtungen erklären, der nach Exstirpation gutartiger Papillome Rückfälle ausgesprochen krebsigen Charakters eintreten sah. Ich selber sah in einem Falle $\left.{ }^{1}\right)$ neben einem unzweifelhaften Medullarkrebs in der gleichen Blase, durch mehrere Centimeter gesunder Schleimhaut von ihm getrennt, ein papilläres Fibrom, welches ich, nach der damaligen Untersuchungsmethode für gutartig hielt. Man wird auch solchen Fällen gegenüber jedenfalls mit der Deutung sehr zurückhaltend sein müssen. Immerhin will ich anführen, dass eine Reihe sehr gewissenhafter Untersucher - Albarran, Schuchardt, Colley, Wendler u. a. - sich unumwunden für die hier erörterte Möglichkeit aussprechen. Ganz neuerdings behauptete Reynès, dass in seinem oben schon erwähnten Falle von Implantation einer "Zottengeschwulst" in der Blase vom Nierenbecken aus einige der multiplen Tumoren als benigne, andere als maligne sich erwiesen. Auch von den ursprünglich gutartigen Geschwülsten der Prostata, wie wir sie muter dem klinischen Bilde der sog. senilen Prostatahypertrophie

1) Ein Fall von primärem Carcinom der Harnblase. Berliner klin. Wochenschrift, 1883 . 
kennen, wird das Gleiche behauptet. Die Gynäkologen verhalten sich in dieser Frage sehr zurïckhaltend.

Ich wïnsche zum Schluss noch Ihre Aufmerksamkeit auf eine theoretisch wie praktisch gleich interessante $\mathrm{klinische} \mathrm{Eigenart} \mathrm{der}$ Blasentumoren zu lenken.

Während die Carcinome des Penis, des Uterus, auch der Prostata sich in Bezug auf die Metastasenbildung nicht viel anders verhalten als anders localisierte Krebse auch, hat der Blasenkrebs eine auffallend geringe Neigung hierzu. Ich habe Fälle dieser Art lange beobachiet, ohne dass eine Spur einer Drüsenanschwellung oder einer sonstigen Metastasenbildung, ja, ohne dass auch nur - solange der Patient nicht durch Blutungen oder durch sekundäre Infektion geschwächt war - die sog. Krebskachexie eintrat. Es ist nicht leicht, diese Tatsache zu deuten; die früher beliebte Erklärung, dass der Mangel an Lymphbahnen in der Blase hieran schuld sei, ist durch Albarrans Untersuchungen widerlegt. Dass sie aber an sich zu Recht besteht, lehrt u. a. auch die Sammelforschung unseres Komitees. Daselbst sind angegeben Metastasen:

unter 773 Fällen von Krebs der Portio 393mal = ca. 50 Proz.

daggegen :

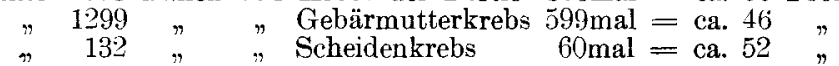

unter 66 Fällen ron Krebs der männl. Blase 15mal =- ca. 23 Proz.

wobei man noch" beriicksichtigen muss, dass unter den letztgenannten Fällen $7 \mathrm{mal}$ bei Männern, 9mal bei Weibern die nächste Nachbarschaft - Mastdarm, Urethra, Prostata, Niere, Uterus, Scheide befallen war, so dass diese Erkrankungen als Kontinuitätscarcinome noch von den "Metastasen" im eigentlichen Sinne abzuziehen wären. Es scheint mir notwendig, diese Eigentümlichkeit zu betonen, damit nicht aus dem Fehlen von Metastasen im Einzelfall Schlüsse auf besonderen klinischen Verlauf oder auf therapeutische Beeinflussung gezogen werden.

M. H. Das hier gegebene Referat kann naturgemäss nur Streiflichter auf die Fülle der Tatsachen werfen und zunächst ohne jede Voreingenommenheit feststellen, an welche Fragen hier die weitere Erforschung des Krebsproblems anzuknüpfen hat. Sie haben gesehen, wie viele und wie mannigfaltige Prozesse bei der Urogenitalcarcinose eine Rolle spielen - ihre Deutung liegt vorläufig nicht in unserer Macht. Auch für unser Gebiet gilt, was B. Fraenkel in seiner mustergültigen Arbeit iiber Metaplasie und Krebs des Kehlkopfes in die treffenden Worte gefasst hat: "W er Zukunftsmusik treiben will, kann über die Registrierung der Befunde hinaus Theorien iiber den ursächlichen Zusammenhang der Prozesse a ufstellen!" 